\title{
Combining Cloud-based Workflow Management System with SOA and CEP to Create Agility in Collaborative Environment
}

\author{
Marian STOICA, Marinela MIRCEA, Bogdan GHILIC-MICU \\ Department of Economic Informatics and Cybernetics, \\ The Bucharest University of Economic Studies, Bucharest, Romania \\ marians@ase.ro,mmircea@ase.ro,ghilic@ase.ro
}

In current economy, technological solutions like cloud computing, service-oriented architecture (SOA) and complex event processing (CEP) are recognized as modern approaches used for increasing the business agility and achieving innovation. The complexity of collaborative business environment raises more and more the need for performant workflow management systems (WfMS) that meet current requirements. Each approach has advantages, but also faces challenges. In this paper we propose a solution for integration of cloud computing with WfMS, SOA and CEP that allows these technologies to complete each other and bank on their benefits to increase agility and reduce the challenges/problems. The paper presents a short introduction in the subject, followed by an analysis of the combination between cloud computing and WfMS and the benefits of cloud based workflow management system. The paper ends with a solution for combining cloud WfMS with SOA and CEP in order to gain business agility and real time collaboration, followed by conclusions and research directions.

Keywords: Cloud Computing, Cloud Workflow Management System, Collaborative Workflow, Workflow-as-a-Service (WaaS), Service Oriented Workflow Management System

\section{1} Introduction

During its evolution, humanity always looked for instruments and innovative solutions that ensure maximum comfort from the perspective of human universe dimensions: familial, social, cultural, economic, religious etc. Many times during this social development, the individual had to resort to various association forms with others in order to achieve these goals, thus laying the foundation for what we call today collaboration. Current forms of association transcend the original goals and the individual aspirations have been altered and transposed into mercantile organization objectives. Unconditional support of these considerations is reflected by the capitalist system and largely by the industrial system, where the aim of earning money through all means outweighs any attempt of collaboration in the original meaning. Unfortunately the mercantilism is not only an economic doctrine of 15 th-18th centuries, but it is still fully manifest in the digital society.

Beyond these considerations, thinking of the social and economic status quo we must notice a change in the business paradigm. The traditional models defined through the goal of maximizing the profit adapt to modern management doctrines, oriented towards the individuals. Current society is not only information and knowledge based; it is also a society of technology and artificial intelligence. Transposing the business in the digital environment is no longer a caprice but a need. Additionally, the simple presence in virtual environment is not the premise for the success of modern business, the advantages provided by technology must also be fully exploited.

Orientation towards exploiting individual abilities and ensuring business processes flexibility has led to what specialists call business agility. Ensuring a certain level of agility for the business requires more and more relying on information and communications technology. Additionally, the agile business status implies redefining the human resource as primary production factor. The anthropocentric paradigm is evidence to this. In the context of modern agile business collaboration and team work are more than ever necessary. Through the prism of dedicated agility technologies, the essence of collaboration as instrument for founding and unfolding economic activities dissolves into the business itself, becoming 
visible on the level of business processes and work tasks. The individuals, activities, processes and work tasks are vertices of informational graphs representing the functioning of modern businesses. The edges of these graphs are merely informational flows (later translated into workflows) that ensure communication and cooperation on business level for reaching its goals.

For technological and economic reasons (meaning resource economy), the need to share resources, skills, competencies, market shares and experience overlays on top of these modern business architecture. In many occasions this was a premise for development of virtual organizations that take advantage of concrete market opportunities. The modern technological solutions is now provided by cloud computing. Together with a workflow management system (WfMS), cloud computing comes to ensure scalability, reutilization of technological capabilities, reliability, improvement of business processes, minimization of information and communications technology (ICT) investment.

Thus, exploitation of cloud services may generate an intensified use of workflows, mainly for collaborative environments. On the other hand, business agility built on service-oriented architecture (SOA) and complex event processing (CEP) solutions may ensure real time collaboration between business processes through processing heterogeneous data extracted from business connected information flows. A solution of workflow management system based on cloud for real time collaboration will provide the capabilities for multi-criterion analyses for business processes, while considering their interdependence with the environment.

In this sense, the paper proposes a complementary approach of cloud computing and workflow management system (WfMS) models in order to identify architectural and/or technological solutions to support real-time collaboration for business processes. After a brief introduction to the field, the proposed work continues with the presentation of an integrated environment defined by these two paradigms, emphasizing the advantages of this integration in collaborative context. Next, the integrated solution is supplemented with tools like SOA and CEP, which support realtime collaboration between processes and reuse of resources. The finality is solution of one technological architecture built to support a new business paradigm in light of CloudWfMS and SOA.

\section{Combining workflow management sys- tems with cloud computing (Cloud WfMS)} Development of information and communication technology, emergence of new business and organization models, increase in resource requirement (networks, servers, storage, services and others), frequent technological changes, as well as using minimal investment to acquire the needed resources make cloud computing a model increasingly widely used. Cloud computing is a technological solution that provides access to virtual sharable resources which may be acquired and released with ease. Also, cloud computing provides benefits like: scalability, reuse of information technology (IT) capability, reliability, improvements for business processes, reduction of IT investment. Intensive and extensive use of cloud services leads to increase in use of workflows, especially in colaborative cloud environments, where there are a lot of heterogeneous resources and complex processes.

At the same time, workflow applications are faced with complex execution environments, variated resources, spread in various geographical areas. Frequent changes require using solutions like cloud computing in order to solve some of the challenges of WfMS. WfMS defines, coordinates, allocates and executes tasks in business processes execution stages. WfMS brings benefits like: metrics, activity audit, improved organization performance, reduced execution time. The interest for this technology is increasing, as reflected both in by literature (books, papers, case studies), as well as in market value ( $\$ 7$ billion by 2018, according to [1]).

\subsection{Cloud computing and workflow man- agement systems}

Cloud computing and workflow management 
are two technological solutions that, used together, complement each other in creating added value. Cloud computing provides benefits to workflow management and at the same time takes advantage of the workflow systems. Even more, there are cases where cloud computing requires a WfMS (for example for easy visual modeling of cloud services) and WfMS requires using cloud computing (for example for easy access to resources).

Cloud computing brings many technological advantages to workflow developers, like: on demand access to software and hardware services, flexibility, origin (source) and reproducibility [2]. Additionally, cloud computing brings the experience of working with workflow managements, gained from grid computing [3]. In cloud environment, the workflow is seen as an emergent technique for scalable applications [4]. The cloud allows users to gain and free resources on demand, which leads to workflow systems that can adapt to changes workflows and availability of resources [5]. Still, we must remember that any technological solution brings both benefits as well as challenges. Therefore cloud computing must be analyzed both from the perspective of benefits to workflow management as well as from the perspective of cloud specific challenges.

The increasingly intensive use of cloud computing has made many practitioners to migrate their workflow management systems to cloud. There are several workflow environments described in literature, like: Cloudbus Toolkit, CloudAnalyst, Swin De W-C (Swinburne Decentralized Workflow for Cloud) [6]. The use of cloud by more and more workflow systems as execution environment increases the challenges related to the efficiency of varied workflow management, virtual machines and execution of instances on virtual machines [7].

A key element in workflow management is workflow scheduling. It plays an important role in managing requests and tasks generated by users and cloud clients. Workflow scheduling is one of the important cloud computing problems, which aims to fully execute workflows, while observing QoS (quality of services) requirements like: budget constraints and deadlines [8]. Task scheduling on backend servers is very difficult, due to the large number of computation requests that require various resources for execution [6]. The workflow performance influences the service quality and response time to user requests.

Adopting workflow systems that allow flexible allocation, automated execution and cloud computing task management leads to improvements in cloud service $[9,10]$. The benefits of WfMS for cloud computing may be grouped on two hierarchic levels [11]: abovethe-cloud and in-the-cloud. Above-the-cloud workflow systems bring advantages like modeling and integration of cloud computing processes, cloud selection and flexible service process scheduling, monitoring and management of execution processes of the cloud computing. A few examples of such systems are: Microsoft Biztalk workflow service and IBM Lotuslive [12]. In-the-cloud workflow systems provide a more efficient control of cloud processes, consolidate self-configuration, improve scalability and error tolerance of the cloud system, reduce operational costs and optimize node communication. One example of such system is Cordys Process Factory (for Google Apps) [12].

Combined use of cloud computing and WfMS can be found in literature as various paradigms like: cloud based workflow management system (Cloud-WfMS), cloud workflow management system, cloud computing oriented workflow management system.

\subsection{Cloud-based workflow management system}

A cloud workflow contains the tasks executed by a participant (individual) or by a service (cloud), a control flow that specifies the order of execution for the tasks and a data flow that specifies the inputs and outputs for the tasks [13]. Cloud WfMS allows modeling and redesigning e-business and e-society processes as cloud workflow specifications (definitions of tasks and QoS nonfunctional requirements) during the modeling stages of development [14]. At the execution instantiation stage selects and reserves the software and hardware 
cloud services that satisfy the workflow specifications. The selection is done following a search and negotiation with cloud resource brokers [14].

Workflow execution may take place in a single cloud or several ones. Execution of a workflow application in a single cloud may be carried out for workflows with small requirements regarding computation resources and input data [3]. Continuous development of ICT has allowed the creation of new complex organizational models, emergence of Internet of Things solutions, which lead to complex workflows that many times imply the existence of several clouds. In high complexity situations, when a single cloud platform is not enough for the large data volumes and computation resources requirements, multiple cloud workflow environments may be used. These environments execute workflow applications using multiple clouds and provide benefits regarding service quality, economy, and execution capabilities for workflows with specific hardware requirements [3].

In [15] Zhao et al. present an integration environment for a cloud organized on four levels (Figure 1): (1 infrastructure layer, that consists of multiple cloud platforms with servers, network and storage resources; 2 middleware layer, that includes Cloud Resource Manager, Scheduling Management Service and Task Scheduling Frameworks subsystems; 3 service layer, that includes Cloud Workflow Management Service and Workflow Engines subsystems; 4 client layer, that includes Workflow Specification \& Submission and Workflow Presentation \& Visualization subsystems.

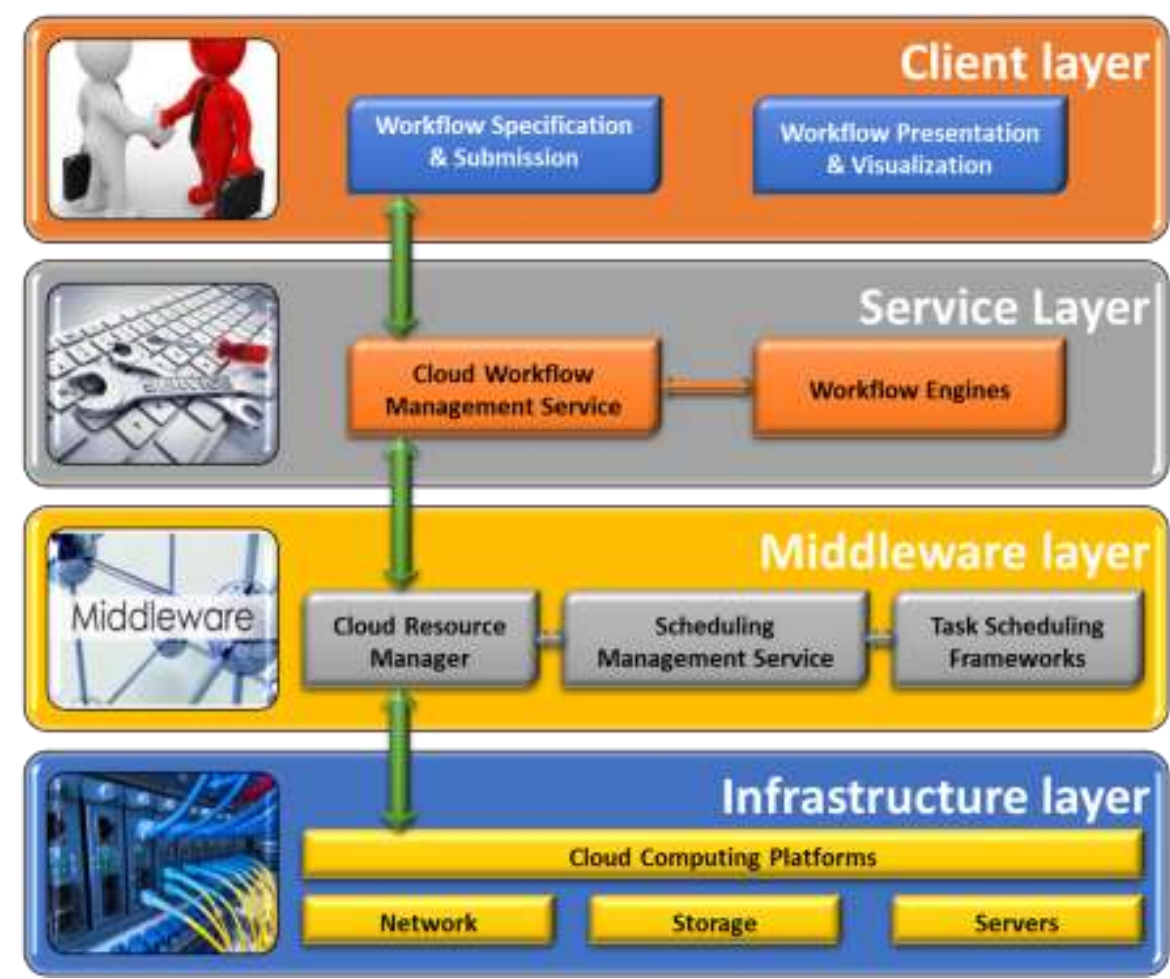

Fig. 1. Cloud-WfMS integration environment (adapted from [15])

During the migration (integration) process towards (into) cloud computing, WfMS may be in of the following situations [16]: ( 0 operational layer in the cloud, 2 task management layer in the cloud, 3 workflow management layer in the cloud, 4 presentation layer or all in the cloud (Figure 2). The first integration happens when the operational level is in the cloud but WfMS still runs outside the cloud. The second level implies that both the operational level and the task management are implemented in the cloud, taking advantage of its facilities. The third integration level means that everything is implemented in the cloud except the presentation level, which is developed as a client virtual machine. The forth 
level assumes that the entire WfMS is implemented in the cloud and can be accesses via a web browser. In the last phase of integration, Cloud-WfMS can provide scalable flows and task management as services (Workflow as a Service - WaaS), thus becoming a kind of SaaS.
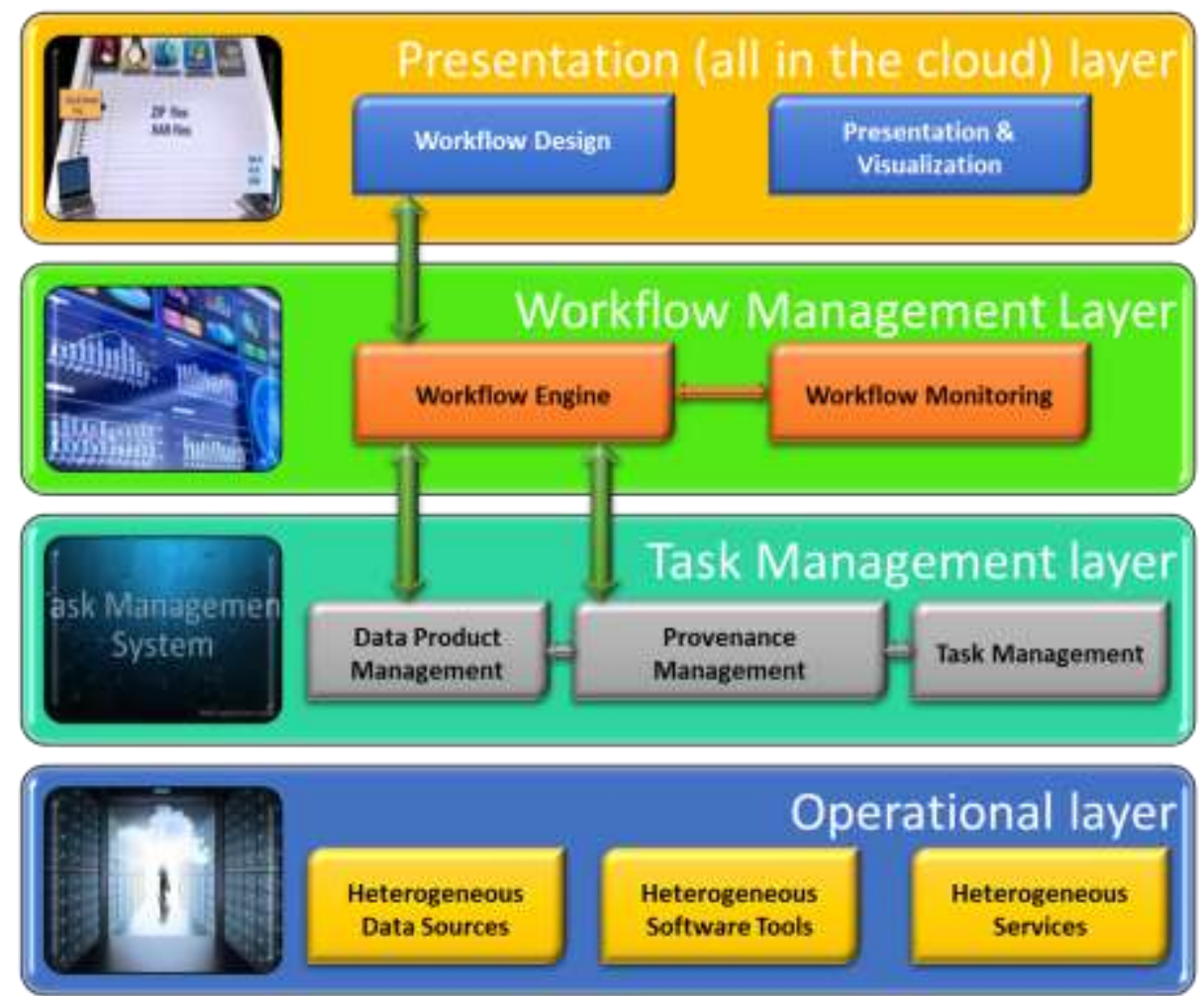

Fig. 2. WfMS cloud computing integration levels (adapted from [16])

Cloud-WfMS takes advantage of the services provided by the cloud infrastructure through SaaS and provides high scalability workflows and task management as services. Among the benefits provided by cloud to workflows are [15]: high computation resources and storage capacities; flexible and comfortable application development; high scalability though a mechanism of on demand resource allocation and final users experience exchange; improved cost/performance ratio through large and varied offer of investment, from dedicated private resources and hybrid resources (local resources and distant cloud resources) to full externalization of computation and storage into public clouds. Also, Cloud-WfMS brings the possibility to share best practice workflows [17], which leads to increased reutilization of work flows.

Still, cloud workflows must face some challenges, like: many participants with various roles involved in creation of workflows, starting task execution, inviting other participants in task execution, adapting workflows, sharing workflow and task prototypes [13].

\section{Combining Cloud-WfMS with SOA and CEP to create agility and real time collabo- ration}

IT solution providers adapted to market demands and large scale use of cloud computing, meeting these demands with cloud based solutions. One of the numerous IT services based on cloud computing is workflow, which can be delivered as a service. The goal of Cloud-WfMS is to provide workflow as a service. One such provider is Nintex, who created in 2016 new cloud solutions for workflow automation platform, including features for delivering Workflow as a service (WaaS) - Nintex Workflow Cloud, Nintex Hawkeye and Nintex Xchange. Also, Nintex has introduced new workflows and provides solutions 
for SharePoint Server 2016 - Nintex for SharePoint 2016 [18]. According to Garner, by 2018 at least $50 \%$ of top providers in enterprise content management will redesign their offer as cloud based platforms [19].

For WaaS to generate real time collaboration and reuse of complex platforms, WfMS architecture must be redesigned to meet some conditions, like: agility, interoperability, scalability and performance. In order to meet the challenges of the complex environment, like Internet of Things, solution architecture must include elements that support solving such problems. The following section analyzes the combined use of Cloud-WfMS, SOA and CEP for real time collaboration and reuse.

\subsection{Cloud-WfMS and Service Oriented Ar- chitecture}

The existence of collaborative environments and fast paced changes force traditional workflow management systems to face difficulties or inflexibility. Service oriented architecture is known to provide loose coupling services (a service cannot call or execute another), services orchestration, remote execution, asynchronous communication, interoperability, service reuse and other benefits that lead to increased agility. The agility is guaranteed by the orchestrator, which is the only service that may invoke other web services. For this reason, web services are loosely coupled and the logic behind collaboration is encoded in the orchestrator [20]. Service orchestrated workflow combines web services with workflow technology to solve integration problems between heterogeneous systems. Also, a WfMS service improves the flexibility, scalability, interoperability and reuse of services and resources thus enabling a more efficient collaboration between applications and other systems.

Real time collaboration for a real business (real time business intelligence, real time business current operations, real time critical decisions or real time business strategies development) anticipating migration of workflows to cloud must be built upon specific enterprise architecture. In other words, to be prepared for the instruments that ensure business flexibility as premise for agility, the information system must provide, including its cybernetic character, perspectives and practices required by service orientation.

Under these considerations, in order to highlight the practical side of supporting real time collaboration through cloud based WfMS, we must go back $~ 10$ years to when EverwareCDBI, company specialized in Service Oriented Application Modernization, launched the meta model for service oriented enterprise architecture: CDBI Service Architecture \& Engineering ${ }^{\mathrm{TM}}$ (CDBI-SAE ${ }^{\mathrm{TM}}$ ) Reference Framework [21]. CDBI-SAE represents a holistic approach defined for SOA, including taxonomies, policies and repeatable processes for designing services in order to achieve business agility. Seen as UML model, the SAE meta model is associated with a diagram of packets describing the way dedicated services, technologies, solution implementation, service specifications, concepts, processes or tasks must be correlated to be able to adapt the model to business requirements [22]. Base on continuous improvement, the reference framework CDBI-SAE has evolved in the last years through adoption of Event Driven Architecture (EDA), Real Time Business Intelligence (RTBI) and cloud based architectural models. From this point of view, the move to Cloud WfMS is natural (figure 3 ) and the representation of the entire business paradigm takes place on three architectural levels:

$\checkmark$ Business architecture (primary information system or operational level);

$\checkmark$ Enterprise architecture (modeling component - associations, attribution, concepts or the analytical-logical level);

$\checkmark$ Cloud-WfMS architecture (processing component or technical-functional level). 


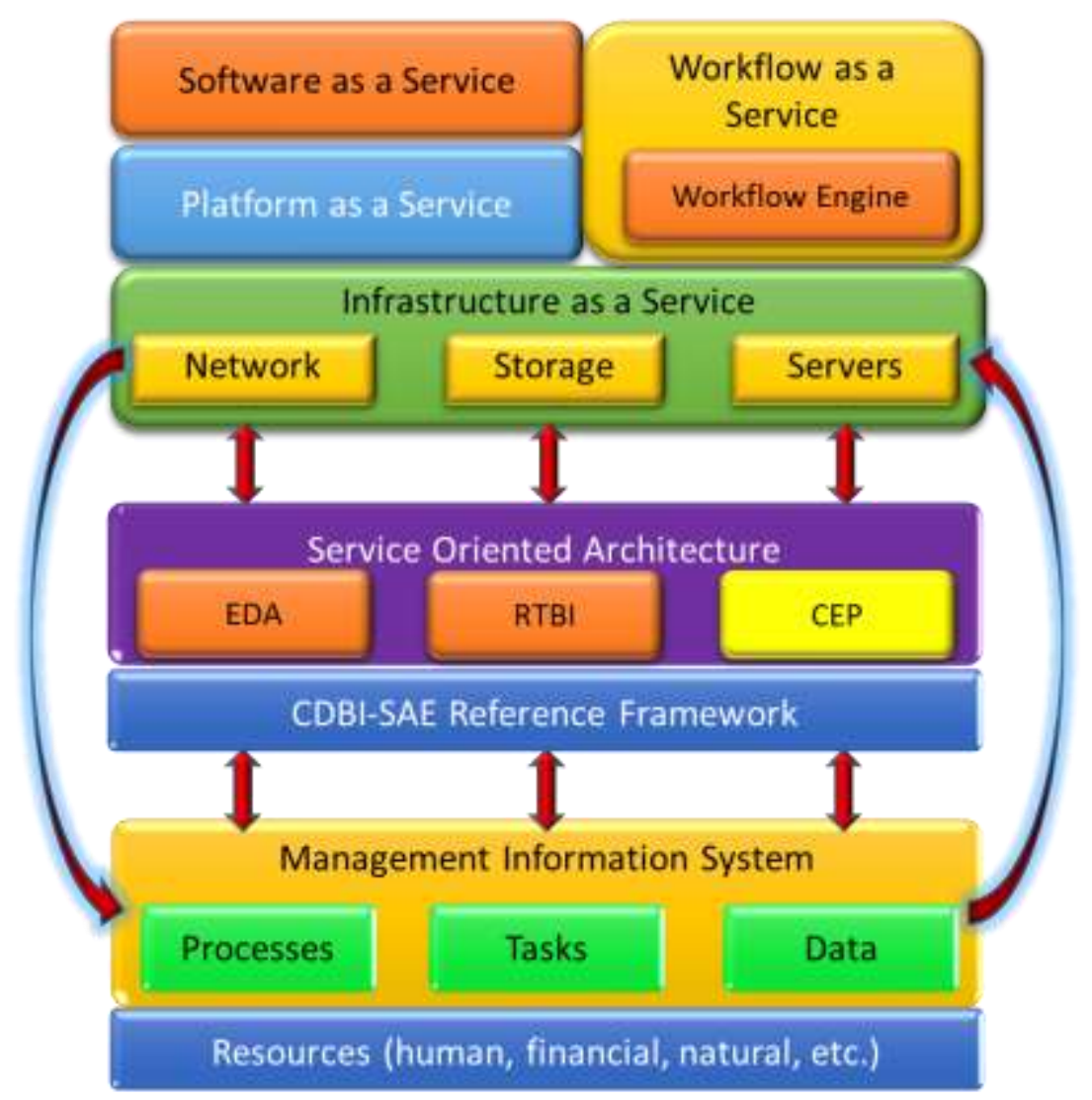

Fig. 3. Business paradigm in light of Cloud WfMS and SOA

In the current economy context, the business environment is faced with a relatively large volume of data, some structured, but mostly unstructured; processing them requires specific instruments. Since SOA does not provide a solution for large volumes of data and the SAE reference architectural frame already adopted EDA and RTBI, the next step in support technology for real time business analyses may be directed towards CEP. Event processing is a method for tracking and analyzing (processing) data generated by certain events. CEP is the event processing that combines varied resources in order to identify models and behaviors of a certain complexity. Thus, SOA and CEP may be considered complementary technologies that mutually amplify their benefits in order to provide collaboration and reuse through real time analysis of data flows. Communication between architectural levels of the new business paradigm is performed through such web dedicated interfaces.

\subsection{Cloud-WfMS and Complex Event Pro- cessing}

Existence of complex collaborative platforms, where large volumes of data (Big Data) come from fast flows, from a wide variety of sources (Internet of Things), may require using CEP. CEP allows real time analysis of data in the information system, as reaction to identification of specific events being triggered (like opportunities or threats in the business lifecycle - see SWOT analysis). Also, it has specialized primitives to collect, analyze and correlate heterogeneous data in order to detect relevant situations in a particular field [23]. In commercial organization context, for example, this field is many times faced with business structural levels, specifically functions like planning, acquisition, sale, marketing, client management etc. In a more analytical context, specific events may be represented as text messages, social media posts, real estate fluctuations, traffic reports, weather reports, exchange rates or other types of data coming from adjacent systems.

In CEP context, an event may be defined as a 
state change, when the level of an indicator goes above a predefined threshold, specific to the field. Organizations that adopt CEP will have a new perspective on the interpretation of economic analytical models, by considering the temporal dimensions in its real behavior state. In other words, modeling business processes can now be done in real time.

In literature, the dominant example invoked in support of CEP advantages is a mechanical system, represented by a vehicle, a set of sensors and a series of events generated by reactions of subcomponents of the technical system. Simplifying for easier understanding, we can associate the behavior model and application of CEP with the thermostat model from thermal systems - on/off reaction to the impulse of a temperature sensor. From this basic example and external complementarity characteristic (see the principle with the same name in the laws of cybernetic systems [25]) manifested by CEP, we can infer the cybernetic characteristic of this paradigm. As consequence, CEP as a business model can be easily placed in a traditional independent context (like traditional organization) or in a
Cloud-WfMS context (like the virtual organization). In any case, the existence of a considerable data flow from various sources (including internet) is implied, and it is the input for the CEP model.

For exemplification we will use a business model from the field of public procurement in Romania, where the authors have significant experience. In a simplified approach, any public procurement takes place in a systemic conference represented (on informational level) by four big actors (providers or beneficiaries of information): Contracting Authority (AC the owner of the procurement procedure main actor), Economic Operators (OE - the pool from which the winner of the procedure is selected), Public Procurement Electronic System (SEAP - web application for brokering public acquisition through electronic means) and the National Agency for Public Procurement (ANAP - public authority for control and monitoring of the procurement procedures). The main flow between these four entities takes place in the cloud and is presented in Figure 4.

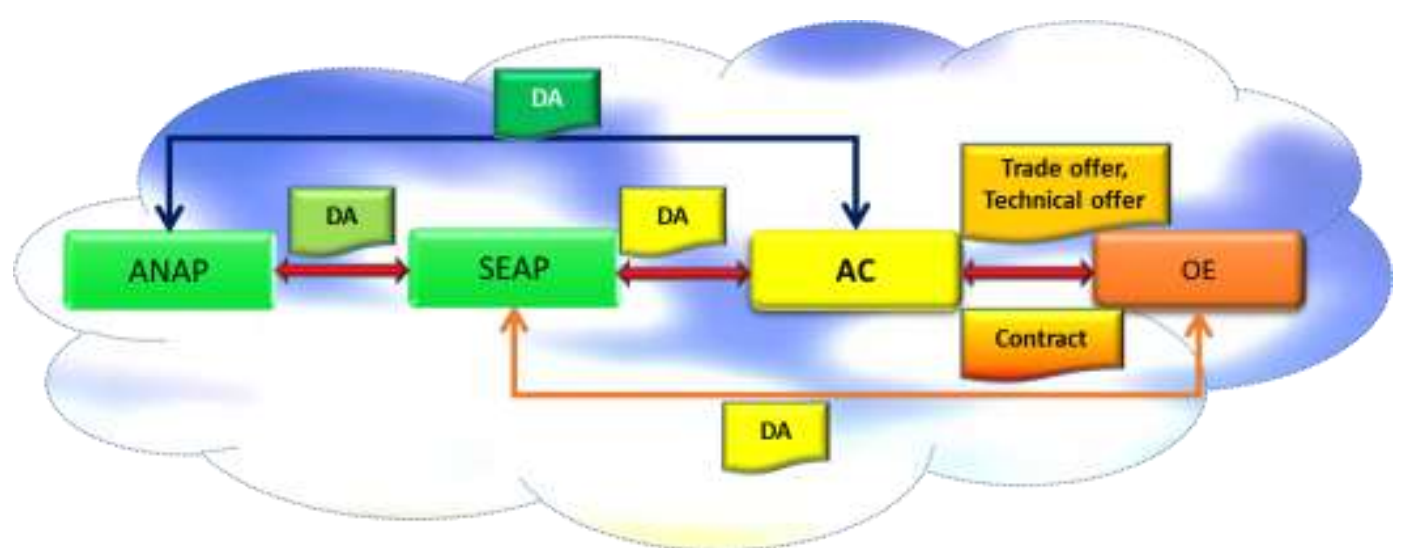

Fig. 4. Cloud-WfMS for procurement procedure

From the perspective of business process management (BPM), the procurement procedure involves at least the following components: centralizing the requests (documents) at AC, establishing financing sources, establishing the economic parameters of the procedure (quantities, prices and quality characteristics), initiating the procedure, completing the tender documentation (DA), publishing the DA, and performing the actual procurement. From CEP perspective there may be events that influence the economic process during any of its components (for example a significant fluctuation of exchange rates or interruption of a financing line - for the establishing the economic parameters stage). In this direction, integration of CEP and BPM must be performed 
at least on two levels: business level (for understanding potential advantages of business processes) and the technological level (there must be a way for CEP to interact with BPM). Public procurement specific workflows will be defined in the AC cloud for own, internal, processes and in interdependence with external information entities in specific virtual environments. When an event that might influence the performing of the procurement process is triggered, the CEP core (software, located in the WaaS component of the AC cloud) will be able to extract the data generated by the event and process them in business representation economic models. This gives the procurement procedure a flexible character, adaptable to interacting events (internal and/or external), generating added value for the beneficiary (AC), by collaboration and real time modeling intermediated by CEP.

\section{Conclusions}

Today it feels like we are going through a new revolution in planning business resources and production factors or, more correctly, that we are main actors in a scenario for significant changes in the way of doing business. Fortunately this is only a feeling, while fundamental models of business are not significantly changed. In the context of this paper we may obviously invoke new production factors in modern organizations, represented by organizational information and culture. Even more, the analytical-scientific endeavor confers the status of neo production factors to technology and business ability to adapt through instruments / solutions like Cloud-WfMS.

The change in the nature of work favored by the spectacular development of information and communication technology has deep implications on the way of doing business. First, a controversial aspect both in practice and literature is the legal dimension of the new forms of work and virtual activities, in general. Cloud technologies are not necessarily novelty elements on the ICT services providers market, they only take a new commercial mantle, an image supported by modern communication infrastructure. Just as well, auto- mation of workflows is not a novelty in business world, but placing it in a cloud context with all its advantages (and disadvantages) coming from this gives them a novelty aura. Considering all these aspects, a Cloud WfMS model for real time collaboration in order to ensure business agility is an integrating solution, both for the information technology elements and the specific components of modern business, taking place mostly though global network. We can notice how collaboration between processes was and remains a solution for business optimization, no matter their context (traditional or virtual).

In conclusion, one of the key elements in business success in the current economic, political and social context is the agile character of the business. There are several ways to gain this attribute for the business, but a hybrid approach like the one proposed in this paper may guarantee it. Built upon the structure of the business information system, the proposed solution must be oriented towards services, calling upon dedicated business architectures. Thus, in order to gain agility independent business features must be harmonized (calling upon BPM), an architecture must be chosen for designing and implementing these features (calling upon SOA) and business decision management and optimization must be ensured (calling upon CEP). The cement between these paradigms is the automated workflows and the infrastructure (the main stage) is the cloud.

\section{References}

[1] Wintergreen. (2012). BPM: market shares, strategy, and forecasts, worldwide, 2012-2018. Lexington: WinterGreen Research. [Online]. Available: http://www.marketresearchre-

ports.biz/analysis-details/business-process-management-bpm-market-sharesstrategies-and-forecasts-worldwide-2012to- 2018

[2] G. Juve and E. Deelman, „Scientific Workflows in the Cloud," Computer Communications and Networks, pp. 71-91, 2011. 
[3] F. Jrad, J. Taom and A Streit. (2013, April 22). A Broker-based Framework for Multi-Cloud Workflows, Prague, Czech Republic. [Online]. Available: https://research.spec.org/icpe_proceedings/2013/multicloud/p61.pdf

[4] M. Bharti and A. Bala. (2012, December). Workflow Management in Cloud Computing. International Journal of Applied Information Systems (IJAIS). [Online]. 4(9). Available: http://research.ijais.org/volume4/number9/ijais12-450788.pdf

[5] R. Buyya, S. Pandey and D. Karunamoorthy, Workflow Engine for Cloud, Cloud Computing: Principles and Paradigms. New Jersey: John Wiley and Sons, 2011.

[6] S. T. Wang, Y. C. Lin and H. Y. Chang, „Developing a Web-Based Workflow Management System in Cloud Computing Platforms," International Journal of Computer, Electrical, Automation, Control and Information Engineering, vol. 9, no. 6, 2015.

[7] J. Wang, P. Korambath, I. Altintas, J. Davis and D. Crawl, ,Workflow as a Service in the Cloud: Architecture and Scheduling Algorithms," Procedia Computer Science, vol. 29, pp. 546-556, 2014.

[8] M. Masdari, S. ValiKardan, Z. Shahi and S. I. Azar, „Towards workflow scheduling in cloud computing: A comprehensive analysis," Journal of Network and Computer Applications, vol. 66, pp. 64-82, 2016.

[9] A. Tsalgatidou, G. Athanasopoulos, M. Pantazoglou, C. Pautasso, T. Heinis, R. Grønmo, Hjørdis Hoff, A. J. Berre, M. Glittum and S. Topouzidou, "Developing scientific workflows from heterogeneous services," SIGMOD Record, vol. 35, no. 2, pp. 2228, June 2006.

[10] C. Hoffa, G. Mehta, T. Freeman, E. Deelman, K. Keahey, B. Berriman and J. Good, "On the use of cloud computing for scientific workflows," in Proceedings of the 2008 Fourth IEEE International Conference on eScience, Indianapolis, Indiana, USA, 2008, pp. 640-645.
[11] H. Huang, Y. L. Zhang and M. Zhang, „A Survey of Cloud Workflow," in Proceedings of the 2nd International Conference On Systems Engineering and Modeling (ICSEM-13), Beijing, China, 2013, pp. 230-236.

[12] J. Zhou, C. Sun,W. Fu, J. Liu, L. Jia, and H. Tan, „Modeling, Design, and Implementation of a Cloud Workflow Engine Based on Aneka," Journal of Applied Mathematics, pp. 1-9, April 2014.

[13] M. Minor, R. Bergmann and S. Görg. (2011). Adaptive Workflow Management in the Cloud-Towards a Novel Platform as a Service. [Online]. Available: http://www.wi2.uni-trier.de/publications/2011_POCBR_MinorEtal.pdf

[14] X. Liu, D. Yuan, G. Zhang, W. Li, D. Cao, Q. He, J. Chen and Y. Yang, The Design of Cloud Workflow Systems. Springer 2012.

[15] Y. Zhao, Y. Li, I. Raicu, C. Lin, W. Tian and R Xue. (2014). Migrating Scientific Workflow Management Systems from the Grid to the Cloud. [Online]. Available:, http://datasys.cs.iit.edu/publications/2014_Cloud4Data_SwiftCloud.pdf

[16] Y. Zhao, Y. Li, I. Raicu, S. Lu, W. Tian and H. Liu. (n.d). Enabling Scalable Scientific Workflow Management in the Cloud. [Online]. Available: https://pdfs.semanticscholar.org/4e6d/4f2bfc1c800b1e 9544 b025aeebf68d172184.pdf

[17] S. Gorg, R. Bergmann, S. Gessinger and M. Minor. (2013). A Resource Model for Cloud-Based Workflow Management Systems Enabling Access Control, Collaboration and Reuse. [Online]. Available: $\quad$ http://www.wi.cs.uni-frankfurt.de/webdav/publications/2013_CLOSER-GoergEtAl.pdf

[18] PRNewswire. (2016, July 06). Nintex to Showcase Workflow as a Service ${ }^{\mathrm{TM}}$ at the 2016. Microsoft Worldwide Partner Conference. [Online]. Available: http://www.prnewswire.com/news-releases/nintex-to-showcase-workflow-as- 
a-service-at-the-2016-microsoft-worldwide-partner-conference-300292278.html

[19] Gartner. (2016, November 7). Gartner Releases 2016 Magic Quadrant for Enterprise Content Management. Gartner. [Online]. Available: http://www.paperfreecorp.com/gartner-releases-2016magic-quadrant-for-enterprise-contentmanagement

[20] Y. Badr, „Service-Oriented Workflow,” Journal of Digital Information Management, vol. 6, no.1, February 2008.

[21] Everware-CBDI. (n.d). CBDI Service Architecture \& Engineering ${ }^{\mathrm{TM}}$ (CBDI$\mathrm{SAE}^{\mathrm{TM}}$ ) Reference Framework, Meta model for SOA. [Online]. Available: http://everware-cbdi.com/products/framework-products/cbdi-sae-metamodel

[22] M. Stoica, C. Bodea, B. Ghilic-Micu and M. Mircea, Managementul sistemelor informaționale. Bucharest: ASE Printing House, 2012, pp. 116-117.

[23] C. Inzinger, W. Hummer, B. Satzger, P. Leitner and S. Dustdar, "Generic eventbased monitoring and adaptation methodology for heterogeneous distributed systems: event-based monitoring and adptation for distributed systems," Software Practice and Experience, vol. 44, no. 7, pp. 805-822, July 2014.

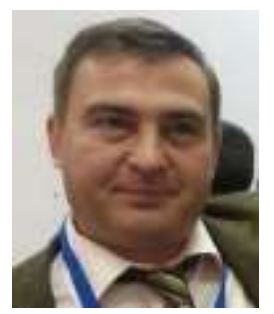

Marian STOICA received his degree on Informatics in Economy from the Bucharest University of Economic Studies in 1997 and his doctoral degree in economics in 2002. Since 1998 he is teaching in Bucharest University of Economic Studies, at Informatics and Cybernetics Economy Department. His research activity, started in 1996 and includes many themes, focused on management information systems, computer programming and information society. The main domains of research activity are Information Society, E-Activities, Tele-Working, and Computer Science. The finality of research activity still today is represented by over 80 articles published, 25 books and over 40 scientific papers presented at national and international conferences. Since 1998, he is member of the research teams in over 30 research contracts with Romanian National Education Ministry and project manager in 5 national research projects.

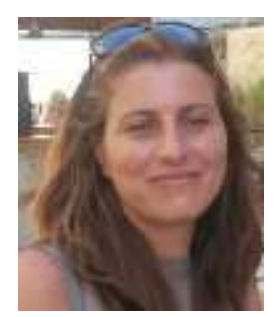

Marinela MIRCEA received her degree on Informatics in Economy from the Academy of Economic Studies, Bucharest in 2003 and his doctoral degree in economics in 2009. Since 2003 she is teaching in Academy of Economic Studies from Bucharest, at Informatics and Cybernetics Economy Department. Her work focuses on the programming, information system, business management and Business Intelligence. She published over 40 articles in journals and magazines in computer science, informatics and business management fields, over 30 papers presented at national and international conferences, symposiums and workshops, she was member over 15 research projects and project manager in 2 national research projects. She is the author and coauthor of 12 books. In February 2009, she finished the doctoral stage, and her $\mathrm{PhD}$ thesis has the title Business management in digital economy.

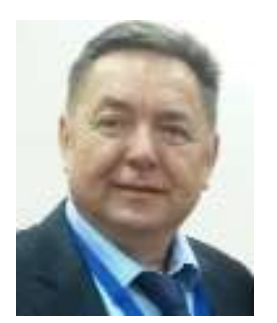

Bogdan GHILIC-MICU received his degree on Informatics in Economy from the Academy of Economic Studies Bucharest in 1984 and his doctoral degree in economics in 1996. Between 1984 and 1990 he worked in Computer Technology Institute from Bucharest as a researcher. Since 1990 he teaches in Academy of Economic Studies from Bucharest, at Informatics in Economy Department. His research activity, started in 1984 includes many themes, like computers programming, software integration and hardware testing. The main domain of his last research activity is the new economy - digital economy in information and 
knowledge society. Since 1998 he managed over 25 research projects like System methodology of distance learning and permanent education, The change and modernize of the economy and society in Romania, E-Romania - an information society for all, Social and environmental impact of new forms of work and activities in information society. 